\title{
DESAFIOS E OPORTUNIDADES PARA O ENSINO REMOTO DA DISCIPLINA DE LABORATÓRIO DE ELETRÔNICA DIGITAL
}

Victor Takashi Hayashi-victor.hayashi@usp.br

Departamento de Computação e Sistemas Digitais da Escola Politécnica da USP Av. Prof. Luciano Gualberto, 158 - Butantã

05508-010 - São Paulo - SP

Felipe Valencia de Almeida - felipe.valencia.almeida@usp.br

Departamento de Computação e Sistemas Digitais da Escola Politécnica da USP Av. Prof. Luciano Gualberto, 158 - Butantã

05508-010 - São Paulo - SP

Reginaldo Arakaki - reginaldo.arakaki@poli.usp.br

Departamento de Computação e Sistemas Digitais da Escola Politécnica da USP Av. Prof. Luciano Gualberto, 158 - Butantã

05508-010 - São Paulo - SP

Edson Toshimi Midorikawa - edson.midorikawa@poli.usp.br

Departamento de Computação e Sistemas Digitais da Escola Politécnica da USP Av. Prof. Luciano Gualberto, 158 - Butantã

05508-010 - São Paulo - SP

Paulo Sergio Cugnasca - cugnasca@usp.br

Departamento de Computação e Sistemas Digitais da Escola Politécnica da USP Av. Prof. Luciano Gualberto, 158 - Butantã

05508-010 - São Paulo - SP

Sergio Roberto de Mello Canovas - sergio.canovas@usp.br

Departamento de Computação e Sistemas Digitais da Escola Politécnica da USP Av. Prof. Luciano Gualberto, 158 - Butantã

05508-010 - São Paulo - SP

Resumo: Este artigo descreve os desafios e oportunidades do ensino a distância para a disciplina de laboratório de eletrônica digital. Através da aplicação de tecnologias de Internet das Coisas, as experiências e projeto dirigido foram readequados para o novo modelo de ensino que respeita as diretrizes de distanciamento social. A solução desenvolvida torna possível a execução do fluxo completo do projeto de sistemas digitais de forma remota: desde o planejamento até a carga do projeto em uma placa FPGA do laboratório. As interações com a placa que eram realizadas de forma presencial foram substituídas por interações via interface mobile, monitoramento através de webcam e kit HomeLab presente na residência do aluno.

Palavras-chave: Laboratório remoto, Arquitetura distribuída, Internet das Coisas. 


\section{INTRODUÇÃO}

Considerando que a grade curricular no curso de Engenharia de Computação da Escola Politécnica da Universidade de São Paulo (Poli-USP) inclui atividades de aprendizado em disciplinas conceituais e de laboratório, este artigo foca nas atividades de laboratório, nas quais a experimentação prática é fundamental para que os conceitos ministrados em disciplinas teóricas sejam percebidos, assimilados e vivenciados na prática. Assim, desenvolvendo a capacitação e habilidade na área da disciplina em questão. Devido a pandemia do Covid-19, o período de isolamento social foi imposto desde março de 2020 no estado de São Paulo, o que dificultou as atividades presenciais, isso criou desafios e oportunidades no processo de ensino e aprendizagem para alunos, professores, técnicos e auxiliares de ensino da instituição.

Os estágios vivenciados desde o início da pandemia impactaram as atividades didáticas do laboratório de eletrônica digital, do primeiro período letivo de 2020, do curso oferecido na modalidade quadrimestral a partir do $3^{\circ}$ ano. Os alunos assistiram uma boa parte das aulas, da ordem de $60 \%$, em laboratório e foi necessário conduzir de maneira virtual os $40 \%$ restantes. Naquele momento, a decisão foi de manter o programa de ensino da disciplina, passando a se usar mais intensamente ferramentas de simulação da implementação. Ou seja, considere-se o ciclo de atividades do aluno nessa disciplina que inclui atividades de (1) projetar, (2) implementar, (3) verificar a implementação, (4) carregar o circuito eletrônico digital em uma placa física conhecida como FPGA (Field Programmable Gate Array), (5) verificar o funcionamento dos circuitos projetados na FPGA, realizando testes e os devidos ajustes em casos de erros observados. Com a pandemia, as atividades (1) e (2) e (3) se mantiveram normais, utilizando-se das ferramentas de projeto já existentes e utilizadas. Já as atividades (4) e (5) foram realizadas por meio de simulações computacionais, e os resultados evidenciados aos docentes por intermédio de reuniões virtuais. Dessa maneira, as atividades que no início do período letivo se iniciaram no laboratório "físico", por conta da pandemia passaram a ser evidenciadas a distância, fortemente lastreadas em simulações.

A grande questão considerada pela equipe foi: como fazer a imersão do aluno no laboratório a distância? Para isso, a iniciativa apresentada neste projeto, que visa permitir a realização de próximos oferecimentos da disciplina a distância, mantendo atividades de implementação física sem lastrear apenas em simulações, considerou a mistura do ambiente físico com o virtual, usando tecnologias de Internet das Coisas (Internet of Things - IoT). Na indústria foi cunhado o termo phygital, segundo Gartner (GARTNER, 2020). Os elementos e papéis do laboratório com características phygital, apresentados neste projeto, são:

- Laboratório de eletrônica digital: entidade física, disponível a distância e dotado de câmeras digitais nas bancadas, conectado na Internet pela plataforma IoT;

- Alunos: estudando e aprendendo em suas casas, fazem a imersão virtual no laboratório digital e realizam ações sobre equipamentos do laboratório;

- Ambiente/moradia do aluno: passa a ser a bancada física onde o aluno utiliza as ferramentas e circuitos físicos de projeto, atuando no que se denomina "bancada HomeLab";

- Professores: ensinando de suas casas, ou eventualmente no laboratório, observam, medem, orientam, auxiliam e avaliam o aproveitamento dos alunos;

- Técnicos de Laboratório: trabalhando de suas casas, ou eventualmente no laboratório, de acordo com as normas de distanciamento social, configuram os equipamentos do laboratório, remota ou presencialmente, e apoiam os alunos nos procedimentos necessários para a realização das experiências; 
- Monitores/Auxiliares de ensino: correspondem a pesquisadores (mestrandos ou doutorandos) que apoiam o desenvolvimento da plataforma IoT;

- Plataforma IoT: elemento que conecta os atores já mencionados, os equipamentos do laboratório e a bancada HomeLab de cada aluno. Tal plataforma oferece uma infraestrutura de comunicação em nuvem pela Internet que permite a implementação do conceito chamado de "imersão", atuando como as "mãos" do aluno, do técnico e do professor nos procedimentos relacionados com os experimentos em laboratório.

Ocorre, então, a combinação do ambiente de laboratório do mundo real com o do mundo virtual (remoto), com experiência de imersão virtual pelas pessoas envolvidas, tanto alunos como professores, técnicos e auxiliares de ensino, formando, assim, a base do phygital. Como sempre ocorreu neste tipo de disciplina de laboratório, o aluno projeta e simula os resultados com as ferramentas computacionais na sua casa. Daí o phygital: uma vez aferido o projeto, ele passa pela jornada de entrar virtualmente no laboratório, mais precisamente na bancada experimental para a qual foi previamente alocado, submete o projeto do circuito desenvolvido e "executa" o resultado obtido, "pressionando" um botão, "acionando" um comando, "visualizando" os leds e displays do aparato experimental por meio de uma webcam, e coleta os resultados, como se estivesse efetivamente presente na bancada do laboratório. Sob a supervisão e apoio dos professores, o aluno evidencia os acertos, os erros, podendo efetuar ajustes, de modo que o ciclo de aprendizado prático se realiza de maneira ampla, completa e quase presencial.

\section{A DISCIPLINA DE LABORATÓRIO DE ELETRÔNICA DIGITAL}

As disciplinas de Laboratório de Eletrônica Digital (I e II) baseiam-se na estrutura de componentes lógicos digitais que incluem portas combinatórias, contadores, somadores, unidades lógicas aritméticas e circuitos de interfaces analógico-digitais para integração com sinais reais do ambiente. A plataforma de implementação é constituída por uma placa de desenvolvimento FPGA, utilizando-se linguagens de descrição de hardware (HDL).

Com este contexto de componentes simples, na primeira disciplina, Laboratório de Eletrônica Digital I, o aluno desenvolve o ciclo de Engenharia: (1) Entender o domínio da aplicação; (2) Especificar os requisitos do projeto; (3) Projetar a solução técnica e os planos de aferição da qualidade que incluem os planos de integração, depuração e testes; (4) Implementar, testar e ajustar o projeto, corrigindo os erros e as discrepâncias nele encontradas; (5) Registrar as atividades, os dados coletados, bem como avaliar e documentar todos os aspectos de Engenharia envolvidos.

Na segunda disciplina, Laboratório de Eletrônica Digital II, todo o ciclo de Engenharia é exercitado mais uma vez, aumentando-se a complexidade dos circuitos compostos por um conjunto de subsistemas. Exercitando o mesmo método, o aluno é desafiado a organizar e treinar em aplicações reais que envolvem mecanismos de transmissão serial, recepção serial, sensores de distância baseados em sonar e atuadores de servo motores controlados digitalmente. Na última versão desta disciplina, estabeleceu-se um domínio de aplicação para engajar os alunos, que deveriam criar e projetar um sistema que utilizasse os subsistemas desenvolvidos na primeira parte da disciplina. Como resultado, foram obtidos excelentes projetos, fortes engajamentos entre os alunos no quesito trabalho em equipe, motivados em concretizar projetos inovadores a partir de uma ideia inicial, ou seja, os alunos foram treinados a assimilar, desde cedo, que inovar exige realizar, executar, corrigir, ajustar. Os resultados foram muito mais do que uma apresentação brilhante de uma ideia implementada somente "no papel". A Figura 1 apresenta a foto de um projeto exemplo desta metodologia, na qual apresenta-se um grupo de 2 
alunos que conceberam e implementaram um jogo utilizando a placa de desenvolvimento FPGA, em conjunto com sonares e servo motores, para implementar um jogo de pinball, com maquetes funcionais para ilustrar os principais requisitos do projeto, que apresentou subsistemas digitais distribuídos e integrados (JUPITERWEB, 2020).

Figura 1 - Projeto Pinball (premiado em $1^{\circ}$ lugar, Laboratório Digital II, em 2019).

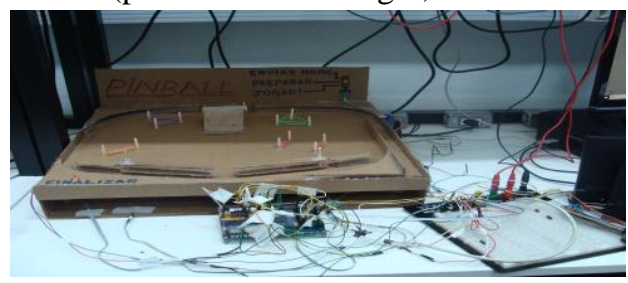

Fonte: Autoria própria.

Com a combinação dos recursos de natureza real e virtual, o desafio didático da disciplina com a pandemia é o de manter esse aspecto da Engenharia, qual seja, o de desenvolver a prática metodológica do ciclo de projeto, implementação e depuração. Como resultado, o planejamento de aulas dirigidas na primeira metade do curso e projeto livre por grupos de alunos foi mantida como evidenciado na Tabela 1. É importante ressaltar que, neste plano, um aspecto é bastante explorado: o da colaboração, pois apesar de os alunos não estarem juntos fisicamente na bancada, devem trabalhar digitalmente juntos, atuando de forma integrada por meio do laboratório phygital.

Os alunos são incentivados a buscar o aprendizado fora do horário de aulas da disciplina. Assim, o departamento oferece a possibilidade dos alunos usufruírem da infraestrutura do laboratório didático em horários alternativos. Este recurso é denominado "OpenLab". A versão para ensino a distância pode ser descrita como um recurso disponível ao aluno fora do horário de aula oferecido remotamente. A mesma infraestrutura usada em horário de aulas pode ser acessada pelos alunos em horários alternativos. Este recurso passa a ser denominado "HomeLab", pois o aluno dispõe de um laboratório didático para seu aprendizado em sua casa.

Tabela 1 - Planejamento das atividades da disciplina de Laboratório de Eletrônica Digital II.

\begin{tabular}{|c|c|c|}
\hline Semana & Atividades / Experiências & Descrição \\
\hline 1 & Experiência o & $\begin{array}{c}\text { Apresentação da disciplina } \\
\text { Introduçẫo ao acesso remoto do laboratório }\end{array}$ \\
\hline 2 & Experiência 1 & Controle de Servo-motor \\
\hline 3 & Experiência 2 & $\begin{array}{c}\text { Sensor ultrassônico de distância HC-SRO4 } \\
\text { Projeto VHDL + Testbenches VHDL + Simulação com ModelSim }\end{array}$ \\
\hline 4 & Experiência 3 & $\begin{array}{l}\text { Transmissão serial básica } \\
\text { Temporização de sinais a partir do Clock e Superamostragem }\end{array}$ \\
\hline 5 & Experiência 4 & Transmissão serial assíncrona com superamostragem \\
\hline 6 & Experiência 5 & Recepção serial assíncrona \\
\hline 7 & Experiência 6 & Projeto base: Sensor de distanciamento social \\
\hline 8 & Projeto 1 & $\begin{array}{l}\text { Entrega: Especificação do projeto do grupo } \\
\text { Apresentação do grupo (Pitch de } 5 \text { minutos) } \\
\text { Desenvolvimento do primeiro módulo }\end{array}$ \\
\hline 9 & Projeto 2 & Entregável 1 \\
\hline 10 & Projeto 3 & Entregável 2 \\
\hline 11 & Projeto 4 & Entregável 3 \\
\hline 12 & Projeto 5 & Entregável 4 \\
\hline 13 & Apresentação do Projeto do Grupo & Apresentação (10 min) + Feira virtual (demonstração) \\
\hline 14 & Recuperação do Projeto & Apresentação e demonstração do projeto \\
\hline
\end{tabular}

Fonte: Autoria própria. 


\section{DESAFIOS DO OFERECIMENTO EAD}

Os desafios enfrentados do laboratório a distância estão descritos na Tabela 2. No geral, o maior destaque é o resultado da combinação do físico com o virtual, ou seja, o aluno não assiste a uma aula prática virtual. Ele executa todas as fases de um ciclo de projeto digital, incluindo as ações de projetar, simular, implementar, testar, ajustar os erros e com isso registrar as experiências e aprendizados obtidos. Conforme esquematizado na tabela, para cada requisito, aspectos da solução e alguns resultados verificados.

Tabela 2 - Mapeamento dos desafios, requisitos, soluções e destaques dos resultados obtidos.

\begin{tabular}{|c|c|c|}
\hline REQUISITO / ATOR & SOLUÇÃO ADOTADA & RESULTADOS OBTIDOS \\
\hline $\begin{array}{l}\text { Laboratório digital } \\
\text { - físico, disponível } \\
\text { a distância. }\end{array}$ & $\begin{array}{l}\text { Conectado pela Internet com } \\
\text { destaques para o uso de câmeras } \\
\text { digitais nas bancadas. }\end{array}$ & $\begin{array}{l}\text { Configuração de ferramentas do } \\
\text { computador da bancada, placa } \\
\text { FPGA e medidores digitais pela } \\
\text { Internet. }\end{array}$ \\
\hline $\begin{array}{l}\text { Alunos - da sua } \\
\text { casa, fazem a } \\
\text { imersão virtual. }\end{array}$ & $\begin{array}{l}\text { O protagonista do laboratório. } \\
\text { Interage com os circuitos locais e } \\
\text { remotos por celular e notebook. }\end{array}$ & $\begin{array}{l}\text { Os alunos são desafiados para } \\
\text { construir projetos loT, pois } \\
\text { conectam circuitos do laboratório e } \\
\text { da casa. }\end{array}$ \\
\hline $\begin{array}{l}\text { Ambiente/moradia } \\
\text { HomeLab- } \\
\text { bancada física. }\end{array}$ & $\begin{array}{l}\text { O laboratório se ampliou. Uma parte } \\
\text { na escola, outra parte na casa, todos } \\
\text { conectados em tempo real. }\end{array}$ & $\begin{array}{l}\text { Combinar o físico com o virtual - } \\
\text { phygital - ampliando a conexão } \\
\text { com o ambiente fora da escola traz } \\
\text { situações reais de projetos. }\end{array}$ \\
\hline $\begin{array}{l}\text { Professores no } \\
\text { ensino de técnicas } \\
\text { digitais. }\end{array}$ & $\begin{array}{l}\text { As conexões digitais permitem que o } \\
\text { professor execute com sucesso as } \\
\text { atividades de observar, medir, } \\
\text { orientar e apoiar os alunos. }\end{array}$ & $\begin{array}{l}\text { Participam e estimulam atividades } \\
\text { na forma de colaboração, muitas } \\
\text { vezes melhor que a interação } \\
\text { convencional. }\end{array}$ \\
\hline $\begin{array}{l}\text { Auxiliares de } \\
\text { ensino (técnicos e } \\
\text { monitores) no } \\
\text { suporte aos } \\
\text { alunos. }\end{array}$ & $\begin{array}{l}\text { A conexão digital entre os } \\
\text { dispositivos, casa e laboratório } \\
\text { permite o acesso ao laboratório e } \\
\text { acesso remoto aos circuitos e } \\
\text { ferramentas. }\end{array}$ & $\begin{array}{l}\text { Monitores e técnicos acessam os } \\
\text { recursos do laboratório e da } \\
\text { bancada HomeLab, } \\
\text { colaborativamente. }\end{array}$ \\
\hline $\begin{array}{l}\text { Plataforma loT } \\
\text { provendo a } \\
\text { infraestrutura de } \\
\text { imersão. }\end{array}$ & $\begin{array}{l}\text { A combinação phygital: circuitos em } \\
\text { casa, circuitos e webcams no } \\
\text { laboratório, smartphones e } \\
\text { computadores dos alunos } \\
\text { digitalmente conectados. }\end{array}$ & $\begin{array}{l}\text { Através do celular ou do } \\
\text { computador em casa, o aluno } \\
\text { "toca" um botão na sua tela que } \\
\text { representa um botão físico. De } \\
\text { maneira similar para visualizar leds, } \\
\text { display e medições dos resultados } \\
\text { dos circuitos. }\end{array}$ \\
\hline
\end{tabular}

Fonte: Autoria própria. 
Com todos esses recursos, os alunos podem construir projetos integrando circuitos sintetizados em FPGA, integrar sensores e atuadores IoT, smartphones, notebooks, leds, displays, fazendo com que os alunos tomem contato com sistemas digitais distribuídos já no $3^{\circ}$ ano de curso.

\section{METODOLOGIA}

Em uma etapa anterior à concepção do projeto, foram estudadas soluções já existentes para viabilizar o oferecimento a distância da disciplina e que poderiam ser utilizadas nesse contexto. Dentre elas, destacam-se o AnyDesk e o Google Meet, que serão descritas a seguir.

O AnyDesk é uma ferramenta que permite a conexão a uma área de trabalho remota, através de um identificador único para cada máquina que o tenha instalado. Essa conexão pode ser estabelecida mediante a aprovação de um administrador ou então apresentando uma senha mestra. Observou-se que o AnyDesk era apresentado como forte candidato ao uso, pois sua interface e estrutura eram de simples uso, pois não depende de configurações adicionais de rede. Outro fator importante é sua disponibilidade em diversos sistemas operacionais diferentes (ANYDESK, 2020).

O Google Meet é um serviço da Google que permite realização de videoconferências, onde um dos participantes pode apresentar sua tela. Nesse contexto, identificou-se no Meet uma possibilidade de viabilizar a apresentação de uma imagem da bancada remota. Isso é possível com o auxílio de webcams conectadas nas bancadas, onde a imagem da câmera é aberta na máquina remota e transmitida aos alunos através da reunião no Meet (GOOGLE, 2020).

Para a criação do conceito de HomeLab foi considerada inicialmente a adoção de soluções prontas como o AnyDesk. Apesar de permitir a realização de todo o procedimento padrão de uso remoto da bancada no laboratório, o AnyDesk se mostrou insuficiente em prover recursos para sincronizar sinais de entrada/saída da FPGA com os componentes eletrônicos localizados na residência dos alunos. Assim, uma solução alternativa foi desenvolvida utilizando tecnologia de ambientes IoT que permitisse a comunicação da placa FPGA, localizada na bancada remota no laboratório, com o HomeLab. A solução escolhida foi utilizar o Blynk.

O Blynk é uma plataforma IoT que permite a comunicação entre dispositivos conectados a um projeto, criado dentro de um aplicativo gratuito em um celular Android ou iOS. Observouse no Blynk uma alternativa viável para o problema da sincronização, sendo que a escolha do mesmo aqui foi mediante a uma análise de adequação ao contexto, onde ressalta-se que outras plataformas IoT também poderiam ser utilizadas (BLYNK, 2020).

Após a escolha da plataforma IoT, foi estabelecido que a conexão entre o aluno e a bancada remota seria realizada apenas por intermédio do Blynk. O AnyDesk então passaria a ser utilizado apenas pelos administradores (professores, técnicos e monitores) ou então por alunos em situações excepcionais. A solução adotada para o acesso remoto do laboratório foi implementar a comunicação aluno-laboratório usando recursos do Blynk, sendo que o feedback visual da bancada remota seria obtido a partir do compartilhamento de tela do Google Meet.

Para a prototipação do projeto, alguns materiais tiveram que ser comprados. Dentre a lista de componentes adquiridos, o mais importante foi o Wemos D1 mini, placa de desenvolvimento do módulo IoT ESP8266. Esse componente é suportado pela plataforma Blynk, e sua comunicação WiFi inclusa permite a comunicação via Internet sem precisar de componentes eletrônicos adicionais. 


\section{PROJETO DESENVOLVIDO}

A Figura 2 fornece a visão geral da solução de laboratório remoto para a disciplina de laboratório de eletrônica digital. Os alunos possuem acesso a interfaces mobile e desktop para acesso aos recursos de videoconferência, planejamento, e execução da experiência. Opcionalmente, possuem um kit HomeLab para realizar montagens de controle e monitoramento com sensores e atuadores (e.g. servo motores e sensores de distância). Através da plataforma IoT, os celulares e kits HomeLab estão conectados ao laboratório digital, onde estão instalados webcam, placa FPGA, computador desktop e kit Lab. Esta conexão é implementada por meio de recursos de videoconferência e plataforma IoT. Para apoio à execução das experiências, um técnico pode estar presente de forma presencial no laboratório, enquanto outros técnicos, professores e monitores podem prestar apoio através de ferramentas de acesso remoto, contribuindo para o distanciamento social inclusive entre a equipe docente.

Na segunda metade do curso, o projeto dirigido terá como temática o contexto pós-covid. Como exemplos, destacam-se projetos para criação de valor para pessoas que moram sozinhas, pessoas com necessidades especiais, com doenças crônicas, ou que facilitem o trabalho colaborativo. Estes sistemas distribuídos podem utilizar a plataforma IoT utilizada durante os experimentos da primeira parte, e conectar componentes presentes no laboratório (FPGA) com componentes das casas dos alunos (kit HomeLab e celular).

Figura 2 - Visão geral da solução desenvolvida.

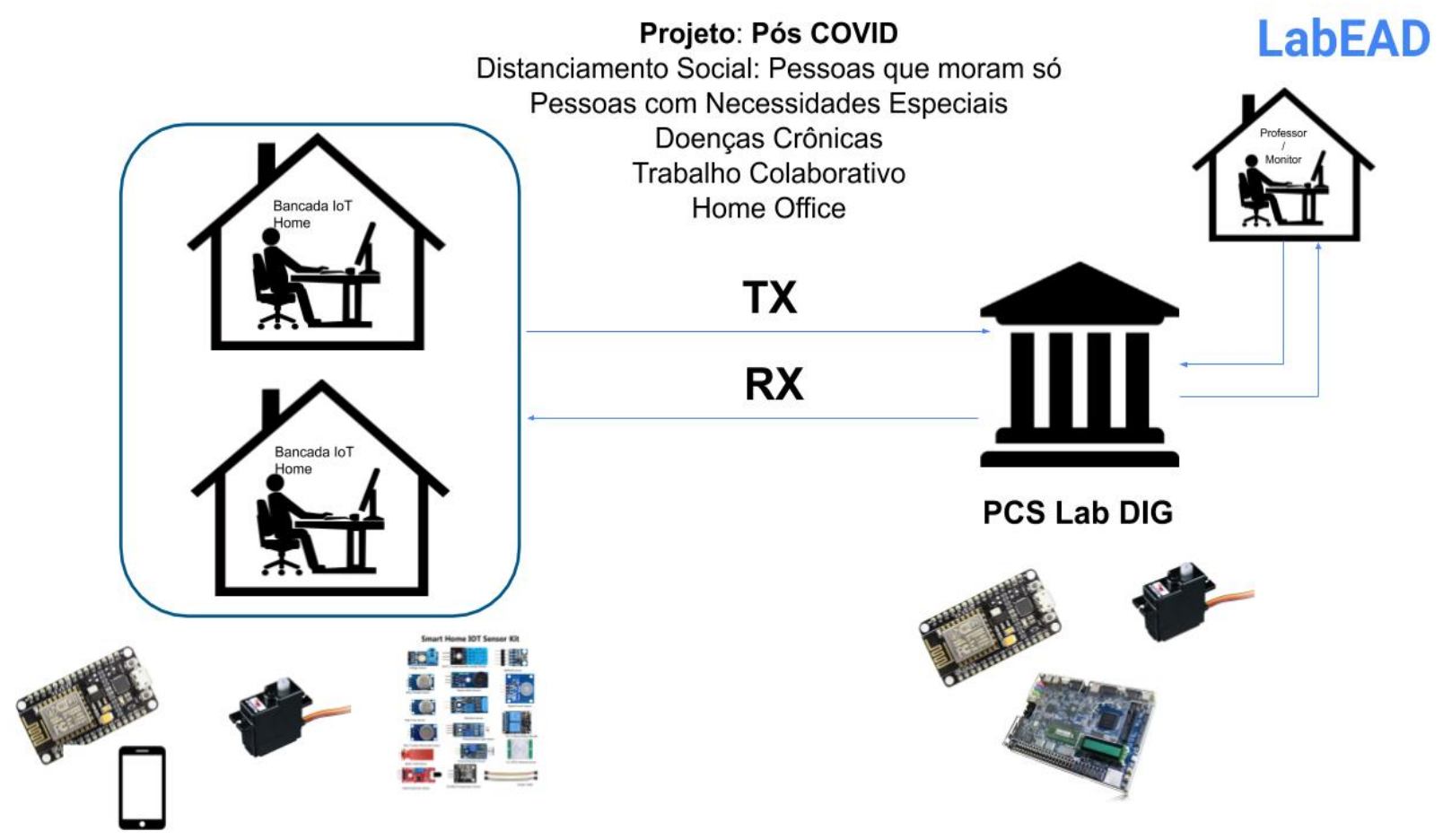

Fonte: Autoria própria 


\section{RESULTADOS}

O sistema de laboratório remoto está sendo implantado na instituição, conforme ilustrado pela Figura 3, para apoiar a disciplina de laboratório de eletrônica digital entre os meses de setembro a dezembro de 2020. São apresentados dois exemplos de uso do laboratório remoto: execução de um experimento da primeira parte do curso, e um projeto simples de um sistema distribuído.

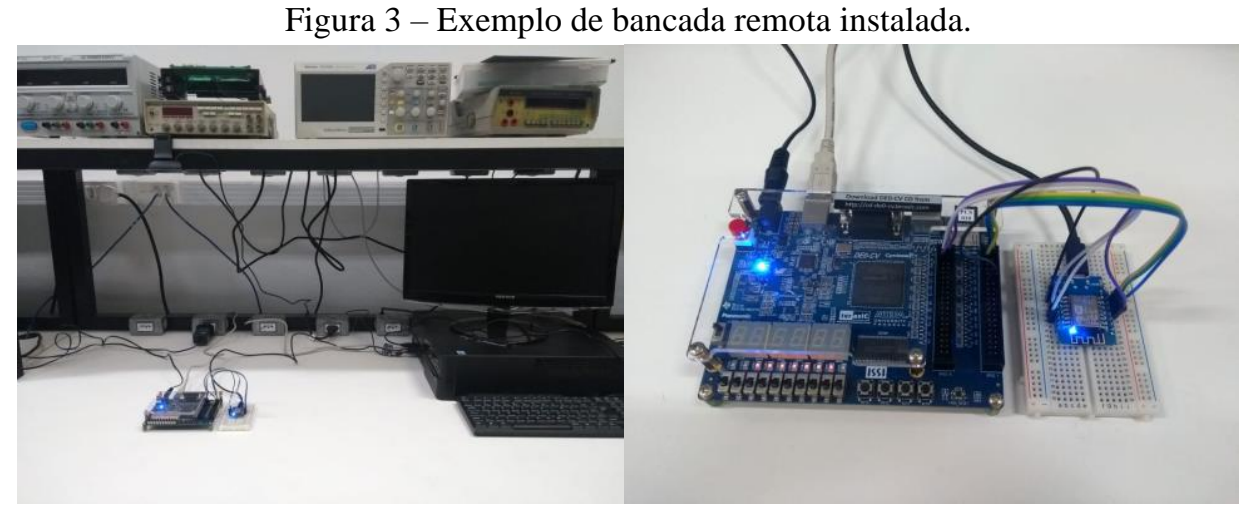

Fonte: Autoria própria

\subsection{EXEMPLO DE EXPERIMENTO: TRANSMISSÃO/RECEPÇÃO SERIAL}

O experimento de transmissão e recepção serial é composto por três etapas. As atividades pré laboratório consistem no projeto do circuito descrito em VHDL com o uso do software Quartus Prime da empresa Intel (antiga Altera), com o mapeamento do pinos e execução de simulações para demonstrar o correto funcionamento do circuito. O projeto operacional é carregado pelo aluno em uma nuvem (Google Drive), com permissão de leitura para o posterior carregamento na placa FPGA. A placa DE0-CV da fabricante Terasic foi utilizada para $\mathrm{o}$ desenvolvimento (INTEL, 2020).

Além do projeto VHDL, o aluno deve ter o aplicativo Blynk instalado no seu celular (iOS ou Android), e criar um projeto com dois terminais: terminal de comandos no pino virtual V0 (terminal CMD), e terminal para a transmissão/recepção de caracteres ASCII no pino virtual V1 (terminal UART). O token de acesso do projeto Blynk do aluno será utilizado a seguir. Testes previstos para o experimento, e questões de entendimento sobre conceitos necessários para a execução do experimento também integram o planejamento.

Durante a execução do experimento na bancada remota, um técnico estará presente no laboratório para realizar manutenções necessárias. A visualização da bancada remota ocorre por meio de videoconferência no Google Meet. Este evento de videoconferência é integrado a evento recorrente na agenda do Google Calendar, e a gestão das sessões fica sob responsabilidade de técnicos, professores e monitores.

O aluno pode comandar a programação do kit Lab com o token de seu projeto através do terminal CMD no seu celular, e comandar o led do kit Lab por meio do mesmo terminal, e verificar o resultado através da videoconferência da bancada remota. Com o identificador do arquivo de seu projeto na nuvem (Google Drive), o aluno também comanda a compilação, mapeamento e carga de seu circuito descrito em VHDL na placa FPGA do laboratório.

Após a execução do experimento na bancada remota, os alunos podem completar o relatório final do experimento com suas análises e resultados obtidos. Caso seja necessário, há a possibilidade de agendamento da bancada remota para que os alunos possam realizar novamente o experimento em horário OpenLab e complementar os resultados. 


\subsection{EXEMPLO DE PROJETO: JOGO DAS TOUPEIRAS}

O jogo das toupeiras é apresentado como um exemplo de projeto dirigido que pode ser implementado na segunda metade do curso. Neste jogo, comumente encontrado em fliperamas, o objetivo é acertar com um martelo a toupeira que aparece de forma aleatória. O jogo foi adaptado para um led multicor e três botões: o jogador deve apertar o botão correspondente à cor que surge de forma aleatória, o que corresponde a atingir uma toupeira com o martelo no jogo original. O sistema está integrado a dispositivos virtuais em um aplicativo (botões, leds), de forma que a interação pode ser feita por meio de celular ou kit HomeLab, conforme Figura 4. Este projeto simples ilustra aspectos de acessibilidade (interfaces mobile e física) e colaboração (um membro do grupo pode jogar com seu kit HomeLab, enquanto o outro pode jogar pelo celular, ao mesmo tempo).
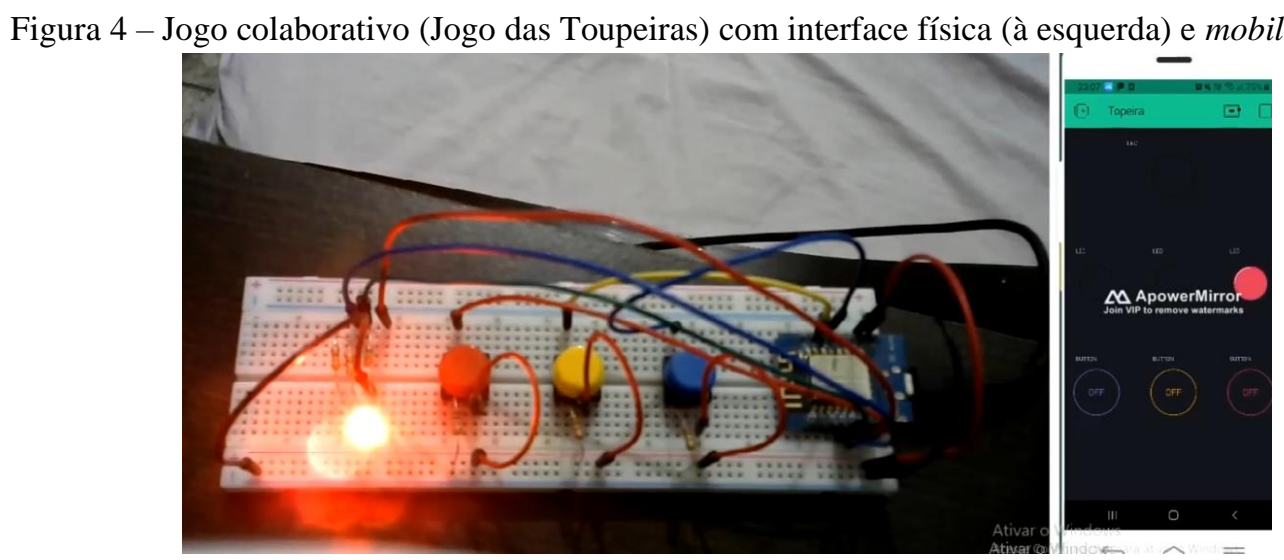

Fonte: Autoria própria

\section{CONSIDERAÇÕES FINAIS}

Este trabalho apresentou o contexto das disciplinas de laboratório de eletrônica digital do curso de Engenharia de Computação da Escola Politécnica da Universidade de São Paulo (Poli/USP), juntamente com os desafios de oferecer disciplinas práticas no momento de quarentena. Estes desafios motivaram a criação de um projeto de laboratório remoto. Observase, nesse cenário, um aumento na oportunidade de estudar projetos voltados ao phygital, sendo que estes terão o potencial de mudar o ambiente de estudo, trabalho e pesquisa em uma sociedade pós Covid-19.

A expansão do projeto relatado para outras disciplinas apresenta-se como oportunidade imediata para trabalhos futuros, visando melhorar o aprendizado dos discentes nas disciplinas práticas curriculares. Além disso, é possível enveredar o projeto para uma implementação corporativa, tornando-o uma infraestrutura para treinamentos remotos.

\section{Agradecimentos}

Agradecemos ao Fundo Patrimonial Amigos da Poli pelo apoio financeiro, e em especial ao técnico de laboratório Daniel Costa Ferreira pela instalação das bancadas remotas no laboratório digital da Escola Politécnica da USP. 


\title{
REFERÊNCIAS
}

\section{Internet:}

ANYDESK. Software para computador remoto. Disponível em: https://anydesk.com/pt. Acesso em: 07 ago. 2020.

BLYNK. Blynk IoT platform: for businesses and developers. Disponível em: https://blynk.io/. Acesso em: 07 ago. 2020.

GARTNER. Fashion Goes Phygital. Disponível em:

https://www.gartner.com/en/marketing/insights/daily-insights/fashion-goes-phygital. Acesso em: 06 ago. 2020.

GOOGLE. Ferramenta de videoconferência Google Meet. Disponível em: https://meet.google.com/. Acesso em: 07 ago. 2020.

INTEL. Altera DE0 Board. Disponível em: https://www.intel.com/content/www/us/en/programmable/solutions/partners/partnerprofile/terasic-inc-/board/altera-de0-board.html. Acesso em: 07 ago. 2020.

JUPITERWEB. Ementa da disciplina de Laboratório Digital II. Disponível em: https://uspdigital.usp.br/jupiterweb/obterDisciplina?sgldis=PCS3645\&codcur=3170\&codhab =3000. Acesso em: 07 ago. 2020 .

\section{CHALLENGES AND OPPORTUNITIES FOR DIGITAL ELECTRONICS REMOTE LAB}

\begin{abstract}
This article describes the remote learning challenges and opportunities for a digital electronics lab course. Given social distancing guidelines, we adapted experiments and planned projects based on top of some Internet of Things technologies. The developed solution lets students perform the complete digital systems project workflow remotely: from planning and simulations to deployment on a physical lab FPGA board. New interfaces, such as mobile interface, webcam monitoring, and HomeLab kit on student's home, replaced face to face interactions between students and FPGA devices.
\end{abstract}

Keywords: Remote lab, Distributed architecture, Internet of Things. 